\title{
Auswirkungen sozialer Belastungen auf das Schul-, Unterrichts- und Arbeitsklima obligatorischer Schulen in der Schweiz
}

\section{Carsten Quesel, Guido Möser und Vera Husfeldt}

Im Rahmen einer schweizerischen Fallstudie wird anhand von Querschnittsdaten der externen Schulevaluation und der Gemeindestatistik des Kantons Aargau überprüft, ob sich die sozialen Rahmenbedingungen signifikant unterschiedlich auf das Schul-, Unterrichts- und Arbeitsklima obligatorischer Schulen auswirken. Zunächst werden die Beziehungen der Konstrukte im Rahmen einer Korrelationsstudie erfasst, hernach wird eine Mehrebenenanalyse durchgeführt, bei der das Unterrichtsklima als abhängige Variable auf der Individualebene fungiert und auf der Aggregationsebene ein Pfadmodell berechnet wird, das sich auf den sozialen Belastungsindex, das Schulklima und das Arbeitsklima erstreckt. Es zeigt sich, dass sich die sozialen Belastungen deutlich auf das Schul-und Arbeitsklima auswirken. Hingegen lässt sich beim Unterrichtsklima keine starke Beeinflussung durch die sozialen Belastungen feststellen. Diese differentielle Wirkung wird darauf zurückgeführt, dass die Lehrpersonen im Unterrichtszimmer eine stärkere pädagogische Wirksamkeit entfalten können als auf der Schulebene.

Schulen sind damit konfrontiert, dass negative soziale Rahmenbedingungen nicht einfach durch das Verschliessen oder Bewachen des Eingangs abgewehrt werden können. Selbst wenn die physische Zugangskontrolle lückenlos wäre, bliebe doch das Problem, dass solche Rahmenbedingungen gleichsam durch das Innenleben der Kinder und Jugendlichen in die Institution hineingetragen werden. Innerhalb der Institution variieren die pädagogischen Chancen, dieses Problem zu bearbeiten, je nach Interaktionsfeld: Im Klassenzimmer ist die Kopplung in der Regel enger als auf den Fluren oder auf dem Pausenhof. Die engere Kopplung im Klassenzimmer stellt indes nicht nur eine pädagogische Chance dar, sondern kann sich auch als massive Beanspruchung auswirken. In diesem Artikel geht es darum, die im Arbeitsklima der Lehrpersonen zum Ausdruck kommenden Beanspruchungen zur Wahrnehmung des Schul- und Unterrichtsklimas auf Seiten der Kinder und Jugendlichen in Beziehung zu setzen. Die Schlüsselfrage lautet, wie sich soziale Umweltbedingungen, die 
durch einen Belastungsindex abgebildet werden, auf diese Aspekte des schulischen Klimas auswirken.

Das Augenmerk dieser Studie konzentriert sich auf obligatorische Schulen in der Schweiz; Grundlage der empirischen Analyse sind Daten der externen Schulevaluation des Kantons Aargau aus dem Zeitraum von 2008 bis 2010 sowie Daten der kantonalen Gemeindestatistik, die über Armut, Arbeitslosigkeit und Zuwanderung Aufschluss geben und zu einem Belastungsindex zusammengefasst werden können. Die Auswertung der Daten erfolgt in zwei Schritten: Zunächst fassen wir explorative Faktorenanalysen in einer Korrelationsstudie zusammen, um dann anhand von deren Befunden eine Mehrebenenanalyse mit einem Pfadmodell durchzuführen.

Die Studie beruht auf 8.662 Fragebögen zum Schul- und Unterrichtsklima aus der Sicht der Kinder und Jugendlichen sowie 3.340 Fragebögen zum Arbeitsklima der Lehrpersonen, verteilt auf insgesamt 108 Gemeinden. Unsere Analyse erfolgt gleichsam aus der «Vogelperspektive»: Wir schauen, ob sich für das Bildungssystem signifikante Befunde ergeben, können uns aber nicht auf Beobachtungen zur Unterrichtsgestaltung stützen. Einschränkend ist zudem darauf hinzuweisen, dass es sich hier um Querschnittsdaten handelt, die es nicht erlauben, Aussagen darüber zu machen, wie sich der Wandel der Rahmenbedingungen auf die Klimafaktoren auswirkt. Eine weitere gewichtige Einschränkung besteht darin, dass im Rahmen dieser Untersuchung die Schulklasse als Aggregationsebene ausser Betracht bleiben muss: Die Daten der externen Schulevaluation können den Schulstufen, Leistungszügen und Gemeinden zugeordnet werden, es ist aber nicht möglich, den Effekt der Klassenzugehörigkeit zu ermitteln.

Im ersten Abschnitt des Artikels sollen aus dem gegenwärtigen Stand der Forschung zu Schulklima und Schulentwicklung die Arbeitshypothesen abgeleitet werden. Der zweite Abschnitt behandelt den methodischen Zugang; darauf folgt jeweils ein Abschnitt zu den empirischen Befunden der Korrelationsstudie und der Mehrebenenanalyse. Auf die Diskussion der Befunde im fünften Abschnitt folgt ein kurzes Fazit.

\section{Soziales Umfeld und schulische KLimafaktoren}

In einem der ersten amerikanischen Handbücher zum Schulmanagement streicht Perry (1908) heraus, dass der "school spirit» wesentlich durch den esprit de corps innerhalb der Schülerschaft geprägt werde: Eine Metapher aus der Mechanik benutzend, rät Perry dazu, diesen Geist als pädagogischen Hebel zu verwenden, um Effizienz und "good government» in der Schule durchzusetzen (Perry, 1908, S. 304). Steht hier die Vorstellung im Hintergrund, dass Schulleitung und Lehrpersonen das Klima in der Schule bestimmen, setzt sich im weiteren Verlauf der Schulklimaforschung der Gedanke durch, dass Schulleitung und Lehrpersonen zwar einen gewichtigen Einfluss auf dessen Entwicklung 
haben, aber nicht in der Lage sind, das Wetter gleichsam aus einer olympischen Position von oben herab allein machen zu können: Sie sind ein Teil des Geschehens und haben dabei mit komplexen Dynamiken zu tun, die in mancher Hinsicht widersprüchlich und nicht selten intransparent sind. Die von Halpin und Croft gewählte Analogie, dass das Klima bei Organisationen der Persönlichkeit bei Individuen entspreche (Halpin \& Croft, 1963, S. 1), zielt auf diesen Punkt ab: Individuen können nicht per Dekret von oben herab zur Persönlichkeit geformt werden - und sie können ihre eigene Persönlichkeit nicht nach Belieben umgestalten. Das liegt nicht zuletzt daran, dass das Geschehen auch durch Einflüsse geprägt ist, deren Quellen außerhalb der Schule anzusiedeln sind. Umgangsformen, Beziehungen von Schülerinnen und Schülern untereinander oder zu Lehrpersonen, die Einhaltung von Regeln und andere Aspekte einer Schul- oder Klassengemeinschaft sind nicht unabhängig von der sozialen Zusammensetzung der Schülerschaft.

Einigkeit besteht in der Schulklimaforschung darin, dass es sich beim Schulklima um "weiche» Merkmale sozialer Wirklichkeit handelt, die anhand von formalisierten Strukturen und Prozesse nicht angemessen erfasst werden können (Anderson, 1982; Cohen \& Geier, 2010; Eder, 1996, S. 25-29; Eder, 2001; Good \& Weinstein, 1986; Johnson, Johnson \& Zimmerman, 1996; Zullig, Koopman, Patton \& Ubbes, 2010). Sucht man in der Schulklimaforschung nach einer gemeinsamen konzeptionellen Schnittmenge, dann treten die erlebte Sicherheit, Anerkennung und Herausforderung als Merkmale des Schulklimas hervor. Im Hinblick auf diese drei Merkmale ist belegt, dass sie stark durch die soziale Umgebung der Schule beeinflusst werden (Hoy \& Woolfolk, 1993; LeBlanc, Swisher, Vitaro \& Tremblay, 2006; Stockard \& Mayberry, 1992; Zullig et al., 2010). Unumstritten ist, dass der Aspekt der physischen Sicherheit eine notwendige Bedingung für ein positives Schulklima darstellt (Freiberg, 1998). Mobbing oder physische Gewalt können das Wohlbefinden einzelner Schülerinnen und Schüler und das Klima ganzer Klassen oder Schulen empfindlich stören. Eng verbunden mit diesen Formen von school disorder ist das Problem der sozialen Benachteiligung. In dieser Hinsicht belegen mehrere Studien, dass hier nicht allein das Wohlstandsniveau maßgeblich ist, sondern auch die ethnische Vielfalt und die Stabilität von Familienstrukturen im Einzugsgebiet der Schule (Bryk, Sebring, Allensworth, Luppescu \& Easton, 2010, S. 182f., 188ff.; Kuperminc, Leadbeater, Emmons \& Blatt, 1997).

Soziale Bindungen und gegenseitige Anerkennung spielen für das Schulklima deshalb eine wichtige Rolle, weil sie eventueller physischer oder psychischer Gewalt entgegenwirken und damit den Aspekt der Sicherheit stärken können. Zum einen geht es hier um die Qualität der Bindungen zwischen Lehrpersonen und Lernenden, zum anderen um die Qualität der Beziehungen der Schülerinnen und Schüler untereinander (Keefe, Kelley \& Miller, 1985; Manning \& Saddlemire, 1996; Whitlock, 2006). Neben der sozialen Akzeptanz spielt beim Schulklima auch der Entwicklungsaspekt eine Rolle: Schülerinnen und Schüler 
sollen in der Schule nicht nur behütet werden, sondern sich auch auf eine konstruktive Weise herausgefordert sehen (Epstein \& McPartland, 1976; Kuperminc, Leadbeater \& Blatt, 2001; Sweetland \& Hoy, 2000). An dieser Stelle kommt der Lehrperson bzw. der Schulleitung eine wichtige Rolle zu. Sie kann das Schulund Unterrichtsklima entscheidend mit beeinflussen und sozial bedingte belastende Einflüsse abmildern.

Wiewohl die sozioökonomischen Rahmenbedingungen wichtig sind, weisen nämlich viele Befunde darauf hin, dass sie das Schulklima nicht völlig determinieren, weil deren Innenleben durch eine mehr oder minder lose Kopplung geprägt ist (Weick, 1976; 1982). Schulen mit identischen Rahmenbedingungen können von den Lernenden sehr unterschiedlich im Hinblick auf die gebotene Sicherheit und das Ausmaß von Fairness und Support bewertet werden (Samdal, Nutbeam, Wold \& Kannas, 1998; Steinberg, Allensworth \& Johnson, 2011) und es fehlt nicht an Beispielen dafür, dass das Klima in Schulen mit schwierigen Rahmenbedingungen von den Kindern und Jugendlichen als positiv erlebt wird (Way, Reddy \& Rhodes, 2007; Whitlock, 2006). Schulen können trotz schlechter sozialer Rahmenbedingungen ein positives Klima erzeugen, das durch hohe Erwartungen, starkes teacher commitment und starkes parent involvement geprägt ist (Brown \& Medway, 2007). Nicht minder wichtig ist hier die Kehrseite der Medaille: Unabhängig von den sozialen Rahmenbedingungen äussern sich Schülerinnen und Schüler auf höheren Schulstufen im Vergleich zur Primarschule oft kritischer zum Schul- und Unterrichtsklima (Eder, 2002; Eder \& Haider, 2012) - was zum einen mit höherem Leistungsdruck und zum anderen mit der Adoleszenz in Zusammenhang gebracht werden kann.

Bryk et al. (2010, S. 77) heben hervor, dass die Entwicklung des Schulklimas stark vom Agieren der Schulleitung und vom professionellen Selbstverständnis der Lehrpersonen abhängig sei. Der Verweis auf Milieufaktoren bei einem schlechten Schul- und Unterrichtsklima entlastet nur insofern, als die Ausgangsbedingungen ungünstig sind, dennoch ist es möglich, dass Schulleitung und Lehrkräfte einen Unterschied machen können (Cohen, McCabe, Michelli \& Pickeral, 2009; Gottfredson, Gottfredson, Payne \& Gottfredson, 2005). Ein regulierendes Eingreifen von Lehrpersonen und Schulleitung dürfte jedoch am ehesten im Unterricht wirksam werden, wo die Möglichkeit der Einflussnahme auf Beziehungen und Regeln naturgemäß stärker ist als außerhalb des Klassenraums.

Zu betonen ist im Zusammenhang mit den Aspekten Sicherheit, Anerkennung und Herausforderung ebenfalls, dass diese in einem Spannungsverhältnis zueinander stehen: Eine Lehrperson, die auf physische Sicherheit insistiert, muss gewalttätigen Schülern die Anerkennung entziehen. Wer unterschiedliche Leistungen diagnostiziert, kann die Akteure nicht unterschiedslos anerkennen. Wo sich Herausforderungen stellen, ist immer auch Verunsicherung im Spiel. Damit zeigt sich, dass die Aufgabe der Steuerung von Schul- und Unterrichtsklima für Lehrpersonen und Schulleitungen keineswegs leicht zu erfüllen ist. Dies 
gilt im Besonderen auch deshalb, weil Sicherheit, Anerkennung und Herausforderung für sie selbst und ihre Arbeitszufriedenheit nicht weniger bedeutsam sind als für Kinder und Jugendliche (Böhm-Kasper, 2004; Johnson, Stevens \& Zvoch, 2007; Sutherland, 1994). Unterstützung bei dieser schwierigen Aufgabe erhalten Lehrpersonen idealerweise sowohl durch die Schulleitung als auch durch den Zusammenhalt im Kollegium. Das Arbeitsklima im Kollegium und speziell der Teamgeist der Lehrpersonen erscheint von daher als wichtige Ressource (Fend, 1986, 1998; Goddard, Hoy \& Hoy, 2000; Holtappels \& Voss, 2006). Umgekehrt ist aber davon auszugehen, dass hohe Anstrengungen zum Erhalt eines guten Klassen- und Schulklimas für Lehrpersonen mit einer höheren Belastung und damit einer verminderten Arbeitszufriedenheit einhergehen.

Die dargestellten Beziehungen zwischen sozialer Umgebung, Belastung, Schul- bzw. Unterrichtsklima und Arbeitszufriedenheit der Lehrpersonen fügen sich zu einem Modell zusammen, dessen Annahmen wir im Folgenden anhand empirischer Daten prüfen möchten. Das Modell ist grob wie folgt zu beschreiben (vgl. Abb. 1): Ungünstige soziale Rahmenbedingungen führen in Schulen tendenziell zu einer Belastung des Schul- und Unterrichtsklimas. Das Schulklima ist davon jedoch stärker betroffen als das Unterrichtsklima, da im engeren Kontext des Unterrichts bessere Einflussmöglichkeiten der Lehrpersonen bestehen, einen belastenden Effekt abzumildern. Diese von der Lehrperson übernommene abmildernde Funktion bleibt in der Regel jedoch nicht ohne Nebenwirkungen: Einerseits trägt der pädagogische Erfolg zwar zur Arbeitszufriedenheit bei, andererseits ist die Bewältigung negativer sozialer Einflüsse aber auch mit Stress verbunden.

Speziell beim Unterrichtsklima ist in Rechnung zu stellen, dass curriculare Anforderungen nicht konstant sind: So kann sich die Relation zwischen Fördern und Fordern zum einen je nach Schulstufe und zum anderen je nach Leistungszug anders darstellen. Kritische Einschätzungen von Schülerinnen und Schülern auf weiterführenden Schulen weisen darauf hin, dass diese Jugendlichen den Unterricht relativ stark unter dem Aspekt des Leistungsdrucks wahrnehmen unabhängig davon, dass sie über relativ gute soziale Chancen verfügen.

Neben den Rahmenbedingungen sind mithin die Sozialisationsphase und die curricularen Anforderungen als wichtige Faktoren für die Ausprägung des Unterrichtsklimas zu würdigen. Bei der hier durchgeführten Analyse können die beiden zuletzt genannten Aspekte indes nur am Rande berücksichtigt werden: Unser Blickwinkel beschränkt sich in dieser Hinsicht darauf, das Unterrichtsklima der Primarschule mit der Sekundarstufe I zu vergleichen. Nicht minder wichtig sind zwei weitere Einschränkungen: Die Kompositionseffekte der Schulklassen und die sich daraus ergebenden Gruppendynamiken müssen bei der hier gewählten Makroperspektive ebenso ausgeklammert werden wie die pädagogische Interaktion im Unterricht.

Die Überprüfung der Annahmen des dargestellten Modells orientiert sich an den drei im Folgenden aufgeführten Arbeitshypothesen. 


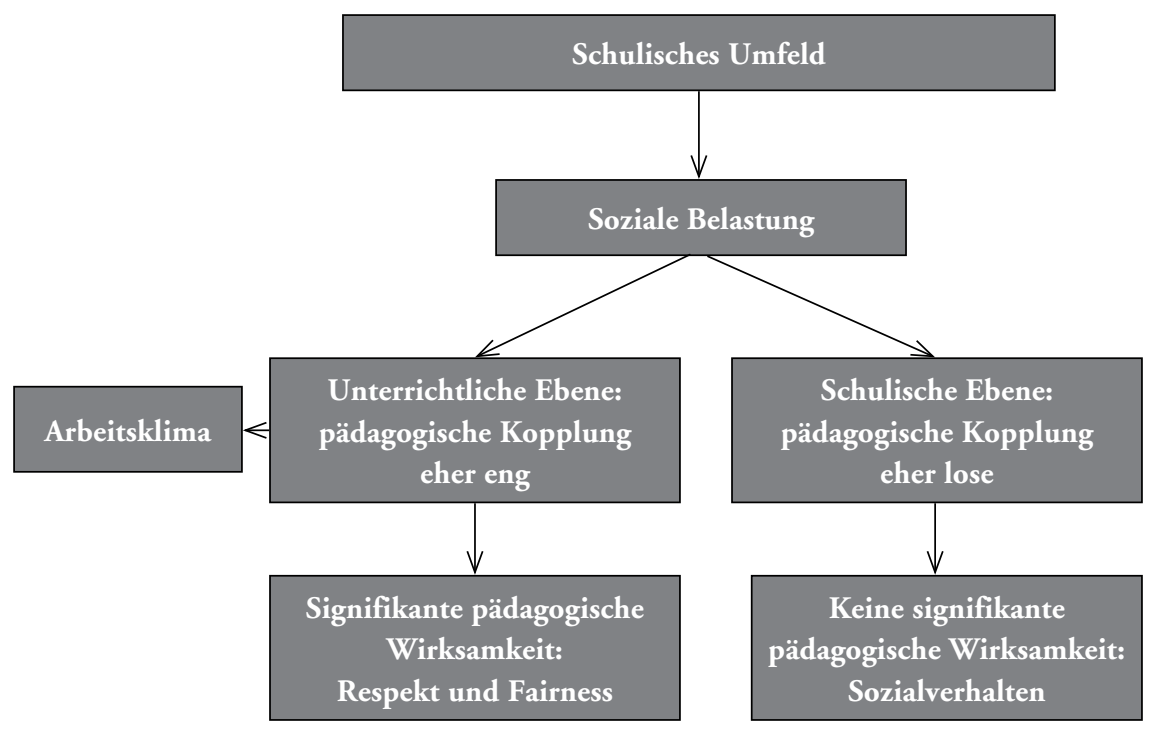

Abbildung 1: Vereinfachte Darstellung der Auswirkungen sozialer Belastungen auf das Schul-, Unterrichts- und Arbeitsklima

1. Hohe soziale Belastungen wirken sich negativ auf das Schulklima und die Rahmenbedingungen pädagogischen Handelns aus.

2. Weil die pädagogische Wirksamkeit der Lehrpersonen auf der Ebene des Unterrichts stärker ist als auf der Ebene der Schule als Organisation, ist das Unterrichtsklima weniger stark durch soziale Belastungen geprägt als das Schulklima.

3. Soziale Belastungen wirken sich negativ auf das Arbeitsklima des Kollegiums aus, es gibt aber keine Kausalkette, die von sozialen Belastungen über negative Ausprägungen des Arbeitsklimas zu negativen Ausprägungen des Unterrichtsklimas führt.

\section{Anlage und Methode der Untersuchung}

Für die Untersuchung zum Zusammenhang von sozialem Einzugsgebiet einerseits und Schul- und Unterrichtsklima sowie Arbeitszufriedenheit andererseits können die Erhebungen der externen Schulevaluation des Kantons Aargau genutzt werden. Zum Zeitpunkt der hier dargestellten Berechnungen konnten zwar nicht alle Schulen berücksichtigt werden, es stehen jedoch umfangreiche und repräsentative Daten aus dem Evaluationszeitraum von Januar 2008 bis September 2010 zum Schul- und Unterrichtsklima sowie zur Arbeitszufriedenheit der Lehrpersonen zur Verfügung. Auf der Basis der gesammelten 
Daten konnten die Fragen aus den verschiedenen Fragebögen zu thematischen Skalen gebündelt werden, wobei die Faktorenanalysen und Reliabilitätstests mit SPSS 22 durchgeführt worden sind. Die für die hier behandelte Fragestellung relevanten Skalen werden weiter unten ausführlicher beschrieben. Das soziale Einzugsgebiet der Schule wurde über den im Kanton Aargau verfügbaren Belastungsindex erfasst. Die Konstrukte werden im Folgenden näher erläutert. Zunächst gehen wir kurz auf die für die Analysen ebenfalls zu berücksichtigende komplexe Schulstruktur ein.

\section{Schuleinheiten}

Die Volksschule des Kantons Aargau erstreckt sich für den hier maßgeblichen Untersuchungszeitraum auf einen zweijährigen Kindergarten, eine fünfjährige Primarschule und eine vierjährige Sekundarstufe I. Die Sekundarstufe I ist unterteilt in die Realschule, die - anders als in Deutschland - den Schultyp mit Grundanforderungen repräsentiert, die Sekundarschule, die einen Schultyp mittleren Anforderungsniveaus bildet, sowie die Bezirksschule, die das höchste Anforderungsniveau verkörpert und als Vorstufe zur gymnasialen Sekundarstufe II dient.

Im Rahmen der externen Schulevaluation werden Schulen evaluiert, die in größeren Gemeinden mehrere Schulhäuser und mehrere Schulstufen unter sich vereinigen, die teilweise in Personalunion von einer Schulleiterin oder einem Schulleiter geführt werden, teilweise aber auch einer Schulhaus- oder Stufenleitung unterstehen. Da nicht nur das Schul- und Unterrichtsklima, sondern auch das Arbeitsklima sowohl zwischen den Stufen als auch zwischen unterschiedlichen Schulhäusern derselben Schule variieren kann, macht es Sinn, in diesem Zusammenhang Schuleinheiten zu betrachten. Sind die Leistungszüge der Real- und Sekundarstufe unter dem Dach desselben Schulhauses untergebracht, werden sie dabei ebenso als getrennte Einheiten angesehen wie mehrere auf eine Gemeinde verteilte Primarschulhäuser. So ergeben sich 149 Schuleinheiten, aus denen zum Zeitpunkt der Untersuchung Daten der externen Schulevaluationen vorliegen. Schuleinheiten, aus denen Daten von je weniger als fünf Personen (Schülerinnen und Schüler oder Lehrpersonen) vorliegen, sind dabei bereits ausgeschlossen und werden wegen ihrer geringeren Aussagekraft nicht mit berücksichtigt.

\section{Belastungsindex}

Jeder der oben beschriebenen Schuleinheiten konnte auf Grundlage der amtlichen Gemeindestatistik ein Belastungsindex zugeordnet werden. Je nach Ausländerquote kann der Index für Quartierschulen auch geringfügig vom Gemeindeindex abweichen.

Die möglichen Werte des Belastungsindexes liegen zwischen 1.00 und 1.40. Ein Belastungsindex von 1 bedeutet, dass die Schule keine zusätzliche Unterstützung benötigt, ein Belastungsindex von 1.4 zeigt eine Unterstützung 
an, die mit dem höchsten Wert, nämlich Faktor 1.4 berechnet wird. Gebildet wird der Belastungsindex, indem die Sozialhilfequote der Wohngemeinde (bezogen auf die Bevölkerung im Alter von fünf bis vierzehn Jahren), die Quote der Einkommensschwachen der Wohngemeinde (gemessen am 25\%-Quartil des Kantons) und die Ausländerquote (ohne Migranten aus Deutschland, Österreich und Liechtenstein) laut Schulstatistik zu einem Index zusammengezählt werden. Dieser Index wird anschließend so transformiert, dass ein Wert mit zwei Nachkommastellen entsteht, so dass also vierzig verschiedene Belastungsniveaus abgebildet werden können. Die Werte des Belastungsindexes sind annähernd normalverteilt mit einem Mittelwert von 1.16 und einer Standardabweichung von .08 (One-Sample-Test nach Kolmogorov-Smirnov: .021, zweiseitig).

Nicht enthalten im Sozialindex sind Daten zu den Familienverhältnissen: Aspekte wie Scheidungsraten oder das Ausmass häuslicher Gewalt können von daher in dieser Analyse nicht berücksichtigt werden.

\section{Schulhaus- und Unterrichtsklima}

Neben dem Belastungsindex konnte den Schuleinheiten je ein Durchschnittswert für die Skalen zum Schul- und Unterrichtsklima sowie zur Arbeitszufriedenheit zugeordnet werden. In Zusammenhang mit der Frage nach normabweichendem Verhalten von Schülerinnen und Schülern sind insbesondere die folgenden zwei Skalen relevant:

- Sozialverhalten SuS (aus dem Schülerfragebogen zum Schulklima)

- Respekt und Fairness (aus dem Schülerfragebogen zum Unterrichtsklima).

Das Schul- und Unterrichtsklima wird auf der Grundlage der Aussagen von Schülerinnen und Schülern gemessen. Um den Befragungsaufwand zu beschränken, wird im Rahmen der externen Evaluation ein Teil der Schülerinnen und Schüler mit der Beantwortung der Fragen zum Schulklima betraut und der andere Teil mit den Fragen zum Unterrichtsklima. Die Aufteilung der Schülerinnen und Schüler geschieht so, dass jeweils ca. die Hälfte einer Klasse den einen Fragebogen bekommt und die andere Hälfte den anderen. Auf diese Weise ist sichergestellt, dass beispielsweise einzelfallspezifische klasseninterne Besonderheiten keine zu große Bedeutung für das Unterrichtsklima in einer Schule bekommen. Trotz dieser Aufsplittung konnten zum Zeitpunkt der hier dargestellten Analysen zu jedem der beiden Klimabereiche Antworten von jeweils knapp 10‘000 Schülerinnen und Schülern erhoben werden. Bei der Beantwortung der Fragen zum Schul- und Unterrichtsklima konnten die Schülerinnen und Schüler zwischen sechs Antwortmöglichkeiten auf Likertskalen wählen, wobei «stimmt gar nicht» den Wert 1 hat und «stimmt genau» den Wert 6.

Die Skalenwerte der Skala Sozialverhalten SuS aus dem Schülerfragebogen zum Schulklima sind Indikatoren für das von den Kindern und Jugendlichen 
erlebte Klima an der Schule als Ganzes. Angst, Bedrohung oder Unwohlsein wird durch geringe Skalenwerte ausgedrückt. Eine weitere Skala zum Thema Respekt und Fairness im Unterricht gibt Auskunft über die Einschätzung der Schülerinnen und Schüler zu Themen wie Respekt, Vertrauen und einem fairen Miteinander im Unterricht. Insgesamt zeigen sich Werte, die im Mittel auf eine hohe Zufriedenheit der Schülerinnen und Schüler mit dem Schul- und Unterrichtsklima schließen lassen (Tabelle 1).

Tabelle 1: Skalen zum Schul-und Unterrichtsklima der Schülerinnen und Schüler

\begin{tabular}{|l|l|l|l|l|l|}
\hline Skala & Beispielitem & $\mathrm{M}$ & $\mathrm{SD}$ & $\mathrm{a}$ & $\mathrm{n}_{\mathrm{i}}$ \\
\hline Sozialverhalten SuS & $\begin{array}{l}\text { Die Schüler/innen gehen an unserer } \\
\text { Schule fair miteinander um. }\end{array}$ & 4.42 & .95 & .75 & 7 \\
\hline $\begin{array}{l}\text { Respekt und Fairness } \\
\text { im Unterricht }\end{array}$ & $\begin{array}{l}\text { In meiner Klasse wird niemand } \\
\text { ausgeschlossen. }\end{array}$ & 4.45 & 1.02 & .79 & 5 \\
\hline
\end{tabular}

$\mathrm{M}$ arithmetischer Mittelwert, SD Standardabweichung, a Cronbachs Alpha, $\mathrm{n}_{\mathrm{i}}$ Anzahl Items

Für die Seite der Lehrpersonen werden insgesamt neun Skalen für die Ermittlung des Arbeitsklimas benutzt. Die Skala Rahmenbedingungen ist dabei auf die Schule als Organisationseinheit bezogen, während die Skala Pädagogische Arbeit sich durch einen starken unterrichtlichen Bezug auszeichnet. Drei weitere Skalen zum Arbeitsklima decken Merkmale der Schulführung ab: es handelt sich um die Skalen Support, Information und Personalentwicklung. Weitere Skalen decken Merkmale des organisatorischen Zusammenhalts ab: Es handelt sich um die Skalen Identifikation, Zusammenarbeit im Team, Kollegiale Beziehungen und Umgang mit Regeln. Um eine bessere Vergleichbarkeit der Skalen herzustellen, wird im Folgenden auf die Skalenmittelwerte Bezug genommen.

Insgesamt sind bei den 3'340 befragten Lehrpersonen hohe Zufriedenheitswerte festzustellen. Auffällig ist bei den Lehrpersonen jedoch, dass bei manchen Fragen sehr homogen und bei anderen sehr heterogen geantwortet wird. Die Einschätzungen zur pädagogischen Arbeit an den Schulen weisen beispielsweise eher geringe Differenzen auf, während die Einschätzungen über Weiterbildungsmöglichkeiten oder darüber, wie gut Lehrpersonen über wichtige Angelegenheiten informiert werden, sehr weit auseinandergehen. Dies geht aus der Betrachtung der Standardabweichungen der Skalenwerte hervor (siehe Tabelle 2). 
Tabelle 2: Skalen zum Arbeitsklima der Lehrpersonen

\begin{tabular}{|c|c|c|c|c|c|}
\hline Skala & Beispielitem & $\mathrm{M}$ & SD & $\mathrm{a}$ & $\mathrm{n}_{\mathrm{i}}$ \\
\hline $\begin{array}{l}\text { Rahmen- } \\
\text { bedingungen }\end{array}$ & $\begin{array}{l}\text { Für die Fächer, die ich unterrichte, ist } \\
\text { diese Schule gut mit Unterrichtsmateri- } \\
\text { alien ausgerüstet. }\end{array}$ & 4.79 & .84 & .79 & 7 \\
\hline $\begin{array}{l}\text { Pädagogische } \\
\text { Arbeit }\end{array}$ & $\begin{array}{l}\text { Von meinen Schülerinnen und Schülern } \\
\text { fühle ich mich akzeptiert und anerkannt }\end{array}$ & 5.03 & .54 & .73 & 5 \\
\hline Führung: Support & $\begin{array}{l}\text { Die Schulleitung beurteilt meine Arbeit } \\
\text { gerecht. }\end{array}$ & 5.00 & .93 & .90 & 8 \\
\hline $\begin{array}{l}\text { Führung: Infor- } \\
\text { mation }\end{array}$ & $\begin{array}{l}\text { Die Schulleitung legt Wert darauf, dass } \\
\text { das Zustandekommen wichtiger Entschei- } \\
\text { dungen für das Kollegium transparent ist. }\end{array}$ & 4.76 & .96 & .91 & 7 \\
\hline $\begin{array}{l}\text { Führung: Perso- } \\
\text { nalentwicklung }\end{array}$ & $\begin{array}{l}\text { An dieser Schule werden die Lehr- } \\
\text { personen gemäss ihren Fähigkeiten } \\
\text { und Interessen gefördert. }\end{array}$ & 4.88 & .98 & .83 & 5 \\
\hline $\begin{array}{l}\text { Kohäsion: } \\
\text { Identifikation }\end{array}$ & Ich glaube, dass dies eine gute Schule ist. & 4.79 & .92 & .89 & 5 \\
\hline $\begin{array}{l}\text { Kohäsion: Zusam- } \\
\text { menarbeit im } \\
\text { Team }\end{array}$ & $\begin{array}{l}\text { Wir helfen uns im Kollegium, bei Schwie- } \\
\text { rigkeiten im Unterricht konstruktive } \\
\text { Lösungen zu finden. }\end{array}$ & 5.01 & .83 & .89 & 7 \\
\hline $\begin{array}{l}\text { Kohäsion: Kolle- } \\
\text { giale Beziehungen }\end{array}$ & $\begin{array}{l}\text { In unserem Kollegium herrscht ein Klima, } \\
\text { in dem unterschiedliche Sichtweisen Platz } \\
\text { haben. }\end{array}$ & 4.98 & .78 & .90 & 8 \\
\hline $\begin{array}{l}\text { Kohäsion: } \\
\text { Umgang mit } \\
\text { Regeln }\end{array}$ & $\begin{array}{l}\text { Die Lehrpersonen setzen sich dafür ein, } \\
\text { dass die vereinbarten Regeln im Alltag } \\
\text { eingehalten werden. }\end{array}$ & 4.89 & .77 & .86 & 4 \\
\hline
\end{tabular}

M arithmetischer Mittelwert, SD Standardabweichung, a Cronbachs Alpha, $\mathrm{n}_{\mathrm{i}}$ Anzahl Items

\section{Zum Vorgehen bei der Datenanalyse}

Nach der oben beschriebenen Skalenbildung wurden die Daten so aggregiert, dass für jede Schuleinheit die Durchschnittswerte für die Skalen des Schul- und Unterrichtsklimas sowie der Arbeitszufriedenheit zur Verfügung stehen. Der aggregierte Datensatz enthält neben diesen Durchschnittswerten ebenfalls den Belastungsindex für jede Schuleinheit. Nach dieser Aufbereitung der Daten ist es möglich, Zusammenhänge zwischen dem Schul- und Unterrichtsklima und der Arbeitszufriedenheit einerseits und dem Belastungsindex andererseits zu analysieren, wobei die untersuchten Einheiten nun nicht mehr die Personen, sondern die Schuleinheiten sind.

Wenn die durch den Belastungsindex ausgedrückte soziale Zusammensetzung der Schülerschaft wie angenommen auf ein bestimmtes Verhalten der Schülerinnen und Schüler in Schule und Unterricht schließen lässt, dann wird sich dies in einem statistisch überprüfbaren Zusammenhang zwischen den Wahrnehmungen zum Sozialverhalten der Schülerinnen und Schüler sowie zu Respekt 
und Fairness im Unterricht auf der einen Seite und dem Belastungsindex auf der anderen Seite manifestieren. Wenn Lehrpersonen es schaffen, in ihren Klassen eine Abmilderung zu erreichen, sollte der Effekt bezüglich des Unterrichtsklimas, hier gemessen mit der Skala Respekt und Fairness im Unterricht, kleiner sein als in Bezug auf das Schulklima (Skala Sozialverhalten). Wenn der Belastungsindex wie angenommen tatsächlich ein Indikator für die Belastung der Lehrpersonen darstellt, dann sollte sich dies in den Aussagen zur Arbeitsbelastung und Arbeitszufriedenheit niederschlagen. Ein höherer Belastungsindex müsste in dem Fall mit einer kritischeren Bewertung der beruflichen Situation zusammenhängen. Um die Zusammenhänge festzustellen, werden Korrelationsanalysen (Produkt-Moment-Korrelation nach Pearson) mit den über die Schulen aggregierten Zufriedenheitswerten und dem Belastungsindex berechnet.

\section{Ergebnisse der Korrelationsstudie}

Wie in der ersten Arbeitshypothese angenommen, lässt sich auf Ebene der Schuleinheiten ein negativer Zusammenhang zwischen dem Belastungsindex und dem anhand des wahrgenommenen Sozialverhaltens bestimmten Schulklima feststellen. Dieser fällt mit $\mathrm{r}=-.33$ hoch signifikant $(\mathrm{p}<0.01)$ aus. Das heißt also, dass in Schuleinheiten, in deren Umfeld eine hohe Quote von Einkommensschwachen, Sozialhilfeempfangenden sowie Migrantinnen und Migranten gezählt wird, tendenziell das Sozialverhalten von den Schülerinnen und Schülern kritischer eingeschätzt wird. Dieser klare Befund deutet darauf hin, dass tatsächlich ein Zusammenhang zwischen den Variablen des Belastungsindexes und einem normabweichenden Verhalten von Kindern und Jugendlichen besteht. Die Items, aus denen die Skala Sozialverhalten SuS zusammengesetzt ist, fragen explizit ab, wie Schülerinnen und Schüler miteinander umgehen und ob dabei psychische oder physische Gewalt eine Rolle spielen.

Für den Unterricht kann diese Beziehung nicht derart deutlich bestätigt werden. Der Grad des fairen Umgangs im Unterricht steht zwar - wiederum auf die Einheit der Schule bezogen - in einem signifikanten Zusammenhang $(\mathrm{r}=-.18 ; \mathrm{p}<0.05)$ mit der sozialen Zusammensetzung des Schulumfeldes, doch ist der Zusammenhang weniger stark als zwischen Sozialverhalten und Belastungsindex. Dieser Befund ist insofern eindrücklich, da er auf eine erfolgreiche Klassenführung der Lehrpersonen in den Schulen mit hohem Belastungsindex hinweist, die offenbar den untersuchten Zusammenhang etwas abzuschwächen vermag. Möglicherweise, so die Annahme, ist dieser Erfolg jedoch mit einer höheren Belastung der Lehrpersonen verbunden.

Um diese Annahme überprüfen zu können, soll an dieser Stelle neben der Einschätzung der Lehrpersonen zu ihrer Belastung auch die empfundene Arbeitszufriedenheit in den einzelnen Bereichen in einen Zusammenhang mit dem Belastungsindex gesetzt werden. Insbesondere die pädagogische Arbeit der 
Lehrpersonen und ihre Zufriedenheit mit den Rahmenbedingungen scheinen stark durch die Belastung in Folge der Schülerzusammensetzung beeinträchtigt zu sein. Aber auch die Einschätzungen zu Personalentwicklungsmöglichkeiten an der Schule oder zum Umgang mit Regeln stehen in einem negativen Zusammenhang zum Belastungsindex. Schließlich sind auch die Identifikation der Lehrpersonen mit ihrer Schule und die Einschätzung zum Support durch die Führung davon betroffen.

Da offenbar die soziale Zusammensetzung der Schülerschaft sich relativ stark in dem von den Schülerinnen und Schülern wahrgenommenen Sozialverhalten niederschlägt, ist es nicht verwunderlich, dass auch die Lehrpersonen je nach Höhe des Belastungsindexes unterschiedliche Wahrnehmungen bezüglich Akzeptanz, Respekt und Entspanntheit des Arbeitsklimas haben. Die Korrelation des Belastungsindex mit der Skala Pädagogische Arbeit ist sehr deutlich negativ und beträgt $\mathrm{r}=-.29(\mathrm{p}<0.01)$. In Schuleinheiten mit einem hohen Belastungsindex scheinen also die Lehrpersonen aufgrund schwierigen Verhaltens der Schülerinnen und Schüler sowie nicht ausreichender Unterstützung seitens der Eltern in ihrer pädagogischen Arbeit beeinträchtigt zu sein.

Der Befund zum Zusammenhang zwischen Belastungsindex und pädagogischer Arbeit legt bei näherer Betrachtung weitere Interpretationen nahe, die mit den anderen Zufriedenheitsbereichen der Lehrpersonen zusammenhängen. Die geringere Zufriedenheit sowohl mit den Rahmenbedingungen als auch mit der Personalentwicklung kommt möglicherweise dadurch zustande, dass die Lehrpersonen an diesen Schulen gerade wegen der Beeinträchtigung ihrer pädagogischen Arbeit einer intensiveren Unterstützung bedürfen. Auf der Grundlage der hier untersuchten Daten kann jedoch nicht eindeutig festgestellt werden, ob ein tatsächlich bestehender Mangel in Ausstattung und Angebot oder ein höherer Anspruch der Lehrpersonen für die geringere Zufriedenheit mit Rahmenbedingungen und Personalentwicklung in Schulen mit hohem Belastungsindex verantwortlich sind. Tatsache ist, dass sich je ein hoch signifikanter $(\mathrm{p}<0.01)$ negativer Zusammenhang zwischen dem Belastungsindex und den mittleren Einschätzungen der Lehrpersonen zu den Rahmenbedingungen ( $\mathrm{r}=-.23)$ sowie $\mathrm{zu}$ den Personalentwicklungsmöglichkeiten ( $\mathrm{r}=-.19)$ feststellen lässt.

Weiter ergibt die Untersuchung der Zusammenhänge, dass Lehrpersonen in Schuleinheiten mit hohem Belastungsindex eher Unzufriedenheit mit den bestehenden Regeln und dem Umgang mit ihnen äußern $(\mathrm{r}=-.17 ; \mathrm{p}<0.05)$ und sich kritischer zum Support durch die Führung verhalten $(r=-.16 ; \mathrm{p}<0.05)$. Auch dies kann vermutlich auf eine schwierigere Situation mit häufigerem Auftreten von normabweichendem Verhalten zurückgeführt werden, in der die bestehenden Regeln möglicherweise als nicht ausreichend angesehen werden und eine stärkere Unterstützung der Schulleitung gefordert wird. 
Tabelle 3: Korrelationen der über die Schuleinheiten aggregierten Skalenwerte mit dem Belastungsindex

\begin{tabular}{|c|c|c|c|}
\hline Befragte & Bereich & Skala & $\begin{array}{l}\text { Korrelation mit } \\
\text { Belastungsindex } \\
\text { (Pearsons } \mathrm{r} \text { ) }\end{array}$ \\
\hline \multirow{2}{*}{$\begin{array}{l}\text { Schülerinnen } \\
\text { und Schüler }\end{array}$} & Schulklima & Sozialverhalten SuS & $-.33^{* *}$ \\
\hline & Unterrichtsklima & Respekt und Fairness & n.s. \\
\hline \multirow{3}{*}{ Lehrpersonen } & \multirow{3}{*}{ Arbeitsklima } & $\begin{array}{l}\text { Rahmenbedingungen } \\
\text { Pädagogische Arbeit }\end{array}$ & $\begin{array}{l}.23^{* *} \\
-.29^{* *}\end{array}$ \\
\hline & & $\begin{array}{l}\text { Support } \\
\text { Information } \\
\text { Personalentwicklung }\end{array}$ & $\begin{array}{l}-.16^{*} \\
\text { n.s. } \\
-.19^{* *}\end{array}$ \\
\hline & & $\begin{array}{l}\text { Identifikation } \\
\text { Zusammenarbeit im Team } \\
\text { Kollegiale Beziehungen } \\
\text { Umgang mit Regeln }\end{array}$ & $\begin{array}{l}-.20^{*} \\
\text { n.s. } \\
\text { n.s. } \\
-.17^{*}\end{array}$ \\
\hline
\end{tabular}

* Korrelation nach Pearson signifikant auf dem 5\%-Niveau.

** Korrelation nach Pearson signifikant auf dem 1\%-Niveau.

Das Miteinander im kollegialen Team der Schule scheint jedoch durch einen hohen Belastungsindex nicht beeinträchtigt zu sein. Die Korrelationen des Belastungsindexes mit den Skalen Führung: Information, Kohäsion: Zusammenarbeit im Team und Kohäsion: Kollegiale Beziehungen sind sämtlich relativ schwach und nicht signifikant ( $>0.05)$. Dennoch scheint die Verbundenheit der Lehrpersonen mit der Schule und die Einschätzung zu ihrer Weiterentwicklung nicht ganz unabhängig von der Zusammensetzung der Schülerschaft zu sein. Die Skala Kohäsion: Identifikation korreliert signifikant $(\mathrm{p}<0.05) \mathrm{mit}$ dem Belastungsindex. Der Korrelationskoeffizient ist $\mathrm{r}=-.18$.

Das Zwischenfazit auf Grundlage der Korrelationsstudie besagt zunächst einmal, dass das Verhalten der Schülerinnen und Schüler und der Umgang miteinander relativ stark mit den im Belastungsindex zusammengefassten Variablen zum sozialen Umfeld der Schule zusammenhängen. Das wirkt sich relativ stark auf das Schulklima und etwas abgemildert auf das Unterrichtsklima aus. Weiter ist ersichtlich, dass auch die Arbeitszufriedenheit der Lehrpersonen in vielen Bereichen davon betroffen ist. Einzig die professionelle Interaktion im Kollegium und mit der Schulleitung scheint davon weitgehend unberührt. Alle Bereiche der Arbeitszufriedenheit, die im engeren oder weiteren Sinne den Umgang mit Schülerinnen und Schülern thematisieren, sind relativ stark durch den sozioökonomischen und soziodemographischen Hintergrund der Schülerschaft beeinflusst. 
Im Hinblick auf die Signifikanz der Korrelationen ist einschränkend darauf hinzuweisen, dass sich die Effektstärken in den meisten Fällen im Spektrum zwischen $r>10$ und $r<.30$ bewegen und insofern gemäß der von Cohen (1988) vorgeschlagenen Konvention als gering zu bewerten sind. Die Ausnahme bildet hier die Korrelation von Belastungsindex und Schulklima, die mit $\mathrm{r}=.33$ eine mittlere Effektstärke aufweist.

\section{Mehrebenenanalyse}

Da sich auf der Ebene der Korrelationen nur erste Hinweise für die in den Arbeitshypothesen postulierten Sachverhalte finden lassen, ist als Vertiefung eine Mehrebenenanalyse durchgeführt worden, die sich an die Verfahrensschritte von Hox (2010) hält. Neben SPSS 22 wurde als Software Mplus 6.1 mit Multilevel Add-On verwendet. In den Datensatz gehen insgesamt 8.662 Fälle (individuelle Ebene) verteilt über 92 Schulen (Aggregationsebene) ein. Fehlende Werte wurden in SPSS mittels EM-Algorithmus geschätzt.

Berechnet wurde ein Zwei-Ebenen-Modell, wobei die Daten zum Unterrichtsklima - bewertet durch die Schülerinnen und Schüler - die abhängige Variable auf der ersten Ebene bilden. Zusätzlich wurden auf dieser Ebene das Geschlecht, der Migrationshintergrund und der besuchte Schultyp mit einbezogen. Der Migrationshintergrund ist durch die Fragen nach dem Geburtsland und die daheim verwendete Sprache operationalisiert worden.

Bei den Schultypen wurde zwischen der Primarschule und den drei Leistungszügen der Sekundarstufe I differenziert; um diese Schultypen in das statistische Modell einbauen zu können, wurden sie jeweils binär codiert. Diese Dummies stellen die unabhängigen Variablen auf der individuellen Ebene dar. (Eine Zuordnung nach Schulklassen ist hier nicht möglich, weil die externe Evaluation ausdrücklich auf die Erfassung dieses Merkmals verzichtet, um den Anspruch zu unterstreichen, dass sie keine Einzelbeurteilung von Lehrpersonen vornimmt.)

Auf der zweiten Ebene sind der Sozialindex und das Arbeitspensum der Lehrpersonen als unabhängige Variablen sowie Schulklima und Arbeitsklima als abhängige Variable angesiedelt. Diese wurden für jede Schule aus der Lehrerbefragung zum Arbeitsklima und der Schülerbefragung zum Schulklima durch Aggregationen in SPSS 22 gebildet, wobei jeweils alle Skalen aus der Korrelationsstudie in die Berechnung der Indizes mit einbezogen wurden: Mithin handelt es sich beim Arbeitsklima um ein second order construct aus neun und beim Schulklima um eines aus zwei Skalen. Abgesehen von Fällen, bei denen ausdrücklich auf Nichtsignifikanz hingewiesen wird, sind alle im Folgenden genannten Parameter mindestens auf dem Niveau von 5\% Irrtumswahrscheinlichkeit (beidseitig) signifikant.

Entsprechend der von Hox (2010) empfohlenen fünfstufigen Vorgehensweise steht das Nullmodell am Anfang der Analyse. Dieses Nullmodell dient 
dazu, die Varianzaufteilung zwischen den Ebenen zu schätzen. Basierend auf diesem Modell kann der ICC berechnet werden, der 12,3 Prozent der Varianz der abhängigen Variablen auf individueller Ebene (Unterrichtsklima) durch die Aggregationsebene (Schule) erklärt. (Läge der ICC nahe beim Nullpunkt, so wäre das ein Hinweis darauf, dass die Unterschiede zwischen den Schulen unbeträchtlich sind und auf die Mehrebenenanalyse verzichtet werden könnte.)

Die nächsten vier Schritte von Hox (2010) bestehen darin, Prädiktoren der beiden Ebenen in das noch leere Modell aufzunehmen. Wir beschränken uns zunächst auf Merkmale der Schülerinnen und Schüler auf Level 1. Bei der Erweiterung des Nullmodells um Geburtsort, Sprache und Geschlecht als Variablen der ersten Ebene mit fixen Effekten (Modell 1) ergibt sich kein inhaltlich relevanter Befund. Gemessen an den standardisierten Regressionskoeffizienten, können sowohl das Geburtsland ( $\beta_{\text {Geburtsland: }}-.014$, nicht signifikant auf 5\%-Niveau), Sprache ( $\beta_{\text {Sprache }}$-.055) und Geschlecht ( $\beta_{\text {Geschlecht: }}$ $.057)$ von der weiteren Analyse aufgrund des niedrigen Einflusses ausgeschlossen werden. Das entspricht der Empfehlung von Hox (2010), um Fehlberechnungen aufgrund von übermässiger Modellkomplexität zu reduzieren.

Die Erweiterung des Nullmodells um die binären Indikatoren für die Schultypen ergibt, dass die Differenzierung nach Schulstufe und Leistungszug einen signifikanten und relevanten Einfluss hat: Die Dummykodierungen in Relation zur Primarschule zeigen für die Leistungszüge der Sekundarstufe 1 mit basalen und mittleren Ansprüchen einen schwach negativen standardisierten Einfluss $\left(\beta_{\text {Sek1_basal }}\right.$-.11, $\beta_{\text {Sek1_ mittel }}$ - -17$)$ und für den Leistungszug mit hohen Ansprüchen einen schwachen bis mittleren negativen standardisierten Einfluss

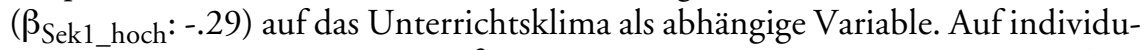
eller Ebene ergibt sich so beim $\mathrm{R}^{2}$ ein Anteil von 6,8\%. Dieser negative Einfluss auf das Unterrichtsklima besagt, dass - gemessen an den Werten für die Primarschule - diese Schulformen ein schlechteres Unterrichtsklima aufweisen.

Auf Level 2 wird im nächsten Schritt ein Pfadmodell umgesetzt, das die Variablen Belastungsindex, Schulklima, Arbeitsklima und Arbeitspensum enthält. Mit diesem Modell soll der Komplexität des schulischen Kontextes in folgender Weise Rechnung getragen werden: Soziale Belastungen wirken sich direkt - etwa in der Form von Mobbing oder Vandalismus - als Stressfaktor auf das Schulklima aus. Lehrpersonen sind teils unmittelbar mit solchen Belastungen konfrontiert und müssen Zeit und Energie aufwenden, um Probleme zu bearbeiten; teils wirken sich diese Probleme aber auch als diffuse «Hintergrundmusik» auf das Arbeitsklima aus. Der Umfang des Pensums könnte dabei in zweierlei Hinsicht bedeutsam sein: Einerseits ist vorstellbar, dass ein höherer Beschäftigungsgrad mit einer stärkeren Beanspruchung einhergeht; andererseits ist es aber auch möglich, dass ein höherer Beschäftigungsgrad eine stärke Identifikation und ein stärkeres Commitment mit sich bringt. Tatsächlich ergibt sich hier kein klarer Befund: Die Variable Arbeitspensum wird aus dem Modell ausgeschlossen, da kein signifikanter Einfluss auf den Achsenabschnitt (im Folgenden Intercept) 
der abhängigen Variable Unterrichtsklima besteht. Einen Überblick über das Pfadmodell liefert Abbildung 2.

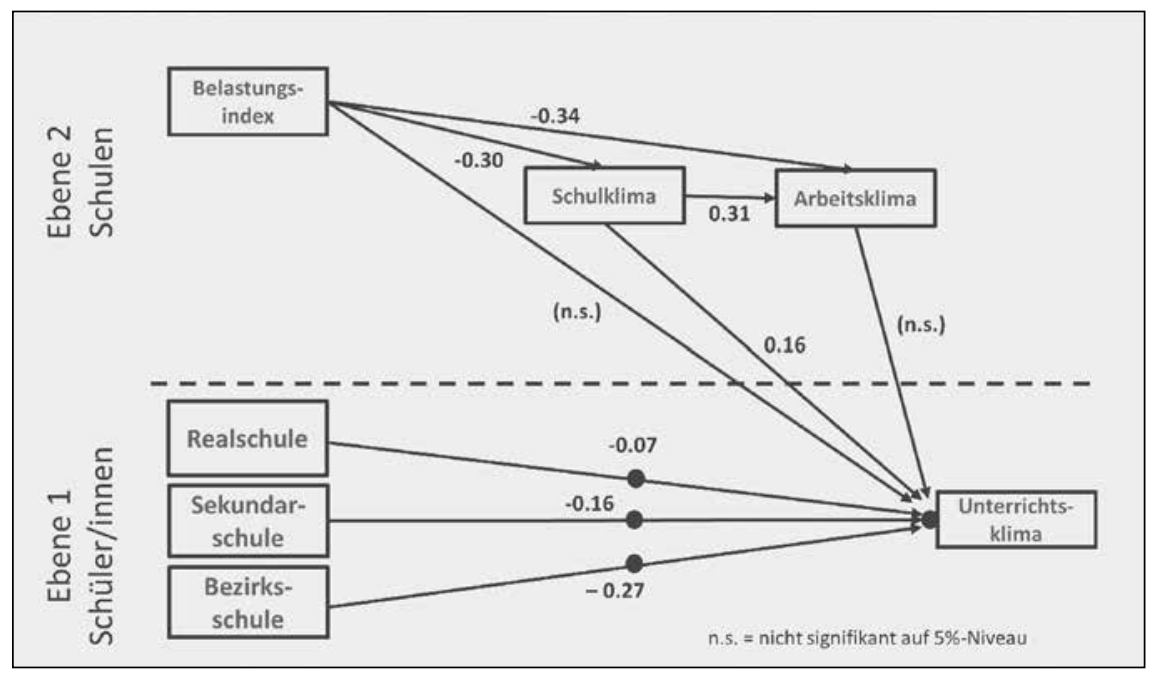

Abbildung 2: Random Slope Modell mit Pfadanalyse auf der Aggregationsebene

Im vierten Schritt werden dann in der Form des Random-Slope-Modells sowohl der Intercept der abhängigen Variable Unterrichtsklima auf individueller Ebene als auch die drei Slopes des Einflusses der unabhängigen Variable Schultyp freigesetzt (Random Intercept und Random Slope). Es wird in diesem Schritt jedoch noch nicht geprüft, ob die Variablen der Aggregationsebene die Variation der Random Slopes erklären.

Hinter der Freisetzung der Konstanten bei der abhängigen Variable Unterrichtsklima steht die Überlegung, dass es sich hier nicht um die Resultante einer deterministischen Beziehung handelt: Zum einen ist mit zufälligen Effekten zu rechnen, die nicht durch die Modellparameter erfasst werden; zum anderen ist davon auszugehen, dass unterschiedliche schulische Kontexte zu unterschiedlichen Niveaus der Ausprägung des Unterrichtsklimas auf der unteren Ebene führen. Die Freisetzung der Regressionsgewichte besagt, dass davon auszugehen ist, dass die im Pfadmodell spezifizierten Variablen auf das Unterrichtsklima so einwirken, dass hier nicht nur unterschiedliche Niveaus bei den Ausprägungen erkennbar sind, sondern der Zusammenhang auch unterschiedlich stark ist. Die sich ergebenden unterschiedlichen Achsenabschnitte und Steigungen sind im Licht der Mehrebenenanalyse nicht als Unschärfe oder Ungenauigkeit zu interpretieren, sondern im Gegenteil als Hinweise darauf, dass die festgestellte Varianz beim Unterrichtsklima systematisch nach Schulstufe und Leistungszug auf der Individualebene und anhand der Elemente und Beziehungen des Pfadmodells auf der Aggregationsebene zerlegt werden kann. 
Der fünfte Schritt des Verfahrens von Hox (2010) zeigt dann, dass keine signifikanten Einflüsse der Variablen auf Aggregationsebene auf die Variation der Random Slopes auf Individualebene zu finden sind. Würden sich hier Koeffizienten ergeben, die signifikant von Null verschieden wären, so hiesse das bei einem positiven Wert, dass der Individualeffekt der Schulzugehörigkeit durch das Pfadmodell verstärkt und bei einem negativen Wert abgeschwächt würde. Eine solche differenzielle Wirkung des Schultyps tritt in den vorliegenden Daten nicht hervor. Weil die Implementation von Cross-Level-Interaktionseffekten im Modell 5 keine Verbesserung des Resultats ergibt, bildet das Modell 4 mit Random Slope, Random Intercepts und mit Pfad-Modell auf Aggregationsebene die Daten am besten ab. Die Varianzaufklärung dieses Modells ergibt auf der individuellen Ebene einen Wert von $\mathrm{R}^{2}=.082$ auf der Aggregationsebene einen Wert von $\mathrm{R}^{2}=.561$.

\section{Tabelle 4: Ausgewählte Kennwerte des Modells 4 im Überblick}

\begin{tabular}{|c|c|c|}
\hline \multicolumn{3}{|l|}{ Fixed part } \\
\hline Predictor & Coefficient & standard error \\
\hline Intercept (Random Intercept) & 1.912 & 0.747 \\
\hline Schulstufe: Sek I basal (Random Slope) & -0.177 & 0.063 \\
\hline Schulstufe: Sek I mittel (Random Slope) & -0.324 & 0.065 \\
\hline Schulstufe: Sek I hoch (Random Slope) & -0.525 & 0.077 \\
\hline $\begin{array}{l}\text { Unterrichtsklima ON } \\
\text { Schulklima } \\
\text { Arbeitsklima } \\
\text { Belastungsindex }\end{array}$ & $\begin{array}{l}0.508 \text { (z: } 0.161) \\
0.056 \text { (z: } 0.017) \\
0.424 \text { (z: } 0.037)\end{array}$ & $\begin{array}{l}0.094 \\
0.078 \text { (n.s.) } \\
0.304 \text { (n.s.) }\end{array}$ \\
\hline $\begin{array}{l}\text { Arbeitsklima ON } \\
\text { Schulklima } \\
\text { Belastungsindex }\end{array}$ & $\begin{array}{c}0.301(\mathrm{z}: 0.313) \\
-1.178(\mathrm{z}:-0.342)\end{array}$ & $\begin{array}{l}0.100 \\
0.358\end{array}$ \\
\hline $\begin{array}{l}\text { Schulklima ON } \\
\text { Belastungsindex }\end{array}$ & $-1.085(\mathrm{z}:-0.302)$ & 0.349 \\
\hline \multicolumn{3}{|l|}{ Random part } \\
\hline$\sigma^{2}{ }_{e}$ & 0.534 & 0.016 \\
\hline $\begin{array}{l}\sigma^{2}{ }_{\mathrm{u} 0}-\text { Schulklima (nicht random) } \\
\sigma_{\mathrm{u} 0}^{2}-\text { Arbeitsklima (nicht random) } \\
\sigma_{\mathrm{u} 0}^{2}-\text { Unterrichtsklima }\end{array}$ & $\begin{array}{l}0.077 \\
0.069 \\
0.036\end{array}$ & $\begin{array}{l}0.010 \\
0.011 \\
0.006\end{array}$ \\
\hline $\begin{array}{l}\mathrm{R}^{2} \text { Individualebene } \\
\mathrm{R}^{2} \text { Aggregationsebene }\end{array}$ & $\begin{array}{l}0.082 \\
0.561\end{array}$ & \\
\hline Deviance & 19.388 & $\mathrm{df}=25$ \\
\hline
\end{tabular}




\section{Diskussion des Mehrebenenmodells}

Die abhängige Variable des hier präsentierten Mehrebenenmodells stellt das Unterrichtsklima auf individueller Ebene dar. Durch Regressionsgleichungen wird für diese Ebene ermittelt, wie stark die unterschiedlichen Schulformen das Unterrichtsklima beeinflussen. Auf der zweiten Ebene wird nun der Einfluss der Gemeindeebene geprüft, wobei als Variablen neben dem Belastungsindex auch das Arbeitsklima und das Schulklima einbezogen werden.

Die Variablen auf der Gemeindeebene können sich auf die Parameter der Regressionsgleichung auf der individuellen Ebene (Einfluss der Schulformen auf das Unterrichtsklima) auswirken. Hier stehen insgesamt vier Parameter der Regressionsgleichung zur Verfügung, die auf einen möglichen Einfluss durch die höheren Ebene und deren Merkmale untersucht werden müssen: Ein Intercept (Achsenabschnitt) und drei Slopes (Steigungen).

Bereits das Nullmodell zeigt, dass der Intercept über die Schulen hinweg signifikant unterschiedlich ist, also eine bedeutsame Varianz aufweist (der im ICC abgebildete Einfluss der Schulen beträgt dabei 12,3 Prozent). In Modell 4 wird nun geprüft, welche Merkmale der höheren Ebene einen Einfluss auf den Intercept zeigen. $\mathrm{Zu}$ diesem Zweck wird der Intercept des Regressionsmodells auf der individuellen Ebene wiederum durch eine Regressionsgleichung ersetzt.

Die Besonderheit des hier entwickelten Mehrebenenmodells besteht nun darin, dass auf der übergeordneten Ebene der Schulen eine Pfadanalyse durchgeführt wird. Diese Pfadanalyse enthält auch Mediationseffekte, so dass zum Beispiel nicht nur die direkte Wirkung des Belastungsindexes auf den Intercept des Unterrichtsklimas beobachtet werden kann, sondern auch die indirekte Wirkung über das Schulklima und das Arbeitsklima.

Zunächst einmal ist im Hinblick auf das Pfadmodell zu konstatieren, dass sich in Entsprechung zu unserer ersten Arbeitshypothese soziale Belastungen in der Tat deutlich negativ auf das Schulklima auswirken. Des Weiteren zeigt sich, dass das Schulklima einen direkten positiven Effekt (standardisierter Koeffizient .16) auf den Random Intercept des Unterrichtsklimas hat - mithin ist der Intercept umso höher, je besser das Schulklima in der Gemeinde ist. Arbeitsklima und Belastungsindex der Schulen (standardisierte Koeffizienten von .02 und .04, beide nicht signifikant) haben jedoch keinen signifikanten direkten Einfluss auf den Random Intercept des Unterrichtsklimas. Die in unserer zweiten Arbeitshypothese ausgedrückte Erwartung, dass das Unterrichtsklima nicht durch die sozialen Rahmenbedingungen determiniert ist, lässt sich insofern bestätigen - allerdings mit der gewichtigen Einschränkung, dass hier keine Entkopplung stattfindet.

Der Belastungsindex zeigt einen mittleren negativen standardisierten Effekt von -.30 auf das Schulklima - das Schulklima ist somit umso schlechter, je höher der Belastungsindex ist. Weil weder ein signifikanter direkter Effekt des Belastungsindex noch des Arbeitsklimas auf das Unterrichtsklima besteht, liegt hier eine totale Mediation des Belastungsindex über das Schulklima auf den Intercept 
des Unterrichtsklimas vor. Wenn es in Schulen gelingt, das Schulklima trotz schwieriger Rahmenbedingungen positiv zu gestalten, wirkt sich das vorteilhaft auf das Unterrichtsklima aus - umgekehrt ist es äusserst schwierig, bei einem negativ ausgeprägten Schulklima ein positives Unterrichtsklima zu kultivieren.

Dieser zuletzt genannte Effekt ist deshalb von Bedeutung, da der Belastungsindex auf Schulebene auch auf das Arbeitsklima mit einem mittleren positiven Effekt wirkt (standardisierter Koeffizient .31). Da sich aber das Arbeitsklima nicht auf den Random Intercept des Unterrichtsklimas auswirkt, erfolgt hier keine Mediation über das Arbeitsklima. Folglich wirkt der Belastungsindex nur indirekt in Form einer totalen Mediation über das Schulklima auf das Unterrichtsklima. Es existieren keine direkten Effekte des Belastungsindex und des Arbeitsklimas auf den Random Intercept des Unterrichtsklimas. Die in unserer dritten Arbeitshypothese formulierte Erwartung, dass das Arbeitsklima keine starken Auswirkungen auf das Unterrichtsklima hat, wird insofern bestätigt - wer als Lehrperson unter schwierigen Bedingungen arbeitet, gibt die erlebte Beanspruchung nicht unweigerlich an die Schülerinnen und Schüler weiter. Einschränkend ist aber darauf hinzuweisen, dass die Daten aus unterschiedlichen Quellen stammen: Das Arbeitsklima nimmt hier einen Sonderstatus ein, weil die Daten von den Lehrpersonen stammen, während sowohl das Schul- wie auch das Unterrichtsklima anhand ein und desselben Schülerfragebogens bestimmt wird.

Auch die drei Random Slopes des Regressionsmodells auf der unteren Ebene werden entsprechend im Mehrebenenmodell durch Regressionsgleichungen ersetzt. Dabei zeigt sich, dass sich die Random Slopes über die Schulen hinweg signifikant unterscheiden. Konkret wirken sich die Schulformen unterschiedlich über die Schulen auf das Unterrichtsklima aus. Ein Einfluss der Merkmale auf der höheren Ebene auf die drei Random Slopes ist aber nicht feststellbar gewesen, so dass Schritt 5 der von Hox (2012) empfohlenen Vorgehensweise hier entfallen kann. Es bestätigt sich für die Schulstufen, dass die Schülerinnen und Schüler auf der Sekundarstufe I unabhängig von den sozialen Rahmenbedingungen tendenziell kritischer über das Unterrichtsklima urteilen. Das kann zum einen mit den wahrgenommenen Leistungsanforderungen und zum anderen mit der Adoleszenz in Zusammenhang gebracht werden, jedoch reichen die hier verwendeten Daten nicht aus, um das im Detail zu belegen.

Die Koeffizienten für das basale, mittlere und höhere Niveau der Sekundarstufe I legen intuitiv den Schluss nahe, dass das Unterrichtsklima systematisch mit dem Leistungszug variiert. Allerdings wäre eine solche Interpretation insofern voreilig, als hier nur auf signifikante Unterschiede zur Primarschule getestet worden ist. Die Differenzen zwischen den Leistungszügen bedürfen noch einer genaueren Untersuchung; ein weiteres wichtiges Desiderat besteht darin, die Arbeitszufriedenheit und die Beanspruchung der Lehrperson detailliert im Hinblick auf die sozialen Kontextbedingungen sowie die Schulstufe und den Schultyp zu untersuchen. Zudem ist einschränkend darauf hinzuweisen, dass die hier verwendeten Querschnittsdaten nicht geeignet sind, die bezeich- 
neten Klimafaktoren mit Sozialisationsverläufen in Verbindung zu bringen. Darüber hinaus ist die Aussagekraft des hier präsentierten Modells limitiert, weil keine Aggregation der Daten auf Ebene der Schulklassen möglich ist: Von daher beschränkt sich das Augenmerk hier auf die Differenz zwischen Schul- und Unterrichtsklima; über die Qualität der Beziehungen in den Klassenzimmern können keine spezifischen Aussagen gemacht werden.

\section{Fazit}

Aus der Analyse der Daten der externen Schulevaluation in Verbindung mit den Informationen zu den gemeindespezifischen Belastungsindices können Antworten auf die anfangs gestellten Fragen abgeleitet werden. Geht man im Sinne der oben geführten Argumentation davon aus, dass die Angaben der Schülerinnen und Schüler zum Sozialverhalten ein valider Indikator für school disorder darstellen, dann kann aufgrund der Ergebnisse konstatiert werden, dass dieses Verhalten in Schulen mit höheren Belastungsindices ein grösseres Problem darstellt als in Schulen mit geringeren Belastungsindices. Die Variablen, die in den Belastungsindex eingehen, also die Kennwerte zu Armut, Migration und Sozialhilfe auf kommunalem Niveau, liefern insofern aussagekräftige Hinweise auf innerschulische Problemlagen. Immerhin gehen elf Prozent der Varianz der pro Schule gemittelten Einschätzungen zum Sozialverhalten auf den Belastungsindex zurück. Jedoch ist dieser Zusammenhang bei Weitem nicht so stark, dass umstandslos von negativen Kontextfaktoren auf das Ausmass problematischen Sozialverhaltens geschlossen werden könnte.

Weiter ist anhand der pro Schule gemittelten Aussagen der Lehrpersonen zu ihrer Arbeitszufriedenheit festzustellen, dass diese in einigen Bereichen relativ stark negativ mit dem Belastungsindex korreliert. Indes liegt hier kein Determinismus vor: Lehrpersonen können schwierige Rahmenbedingungen als Chance nutzen, um ihre Professionalität unter Beweis zu stellen - umgekehrt sind aber günstige Rahmenbedingungen keine Garantie für Erfolg, Anerkennung und Selbstbestätigung. In dieser Hinsicht ist nochmals auf die Limitierungen des von uns gewählten Zugangs aufmerksam zu machen: Bei der hier gewählten statischen Makroperspektive muss das Unterrichtsgeschehen selbst und damit auch das pädagogische Handeln der Lehrpersonen ausser Betracht bleiben. Von daher stellt sich die Aufgabe, soziale Belastungen, Klimafaktoren und pädagogische Interaktion in einer längsschnittlichen Perspektive zu untersuchen. Eine solche Längsschnittperspektive ist zudem deshalb erforderlich, weil der Belastungsindex die Funktion hat, Schulen in schwieriger Lage mit speziellen Ressourcen zu unterstützen, die etwa im Bereich der Sprachförderung oder der Hausaufgabenbetreuung eingesetzt werden können. Bislang lässt sich nicht sagen, wie wirksam dieser politische Steuerungsversuch tatsächlich ist.

Dass die Einschätzungen der Schülerinnen und Schüler zu Respekt und 
Fairness im Unterricht in weniger starkem Zusammenhang mit dem Belastungsindex stehen als ihre Einschätzungen zum Sozialverhalten im Allgemeinen, kann als Indiz dafür verstanden werden, dass es vielen Lehrpersonen in Schulen mit hohem Belastungsindex gelingt, in ihrem Unterricht Regeln des fairen und respektvollen Umgangs durchzusetzen. Gleichwohl nehmen die Schülerinnen und Schüler im Laufe der Adoleszenz unabhängig von der sozialen Belastung eine kritischere Haltung zum Unterrichtsklima ein - wobei das durchaus nicht mit einem pädagogischen Misserfolg gleichzusetzen ist.

\section{Literatur}

Anderson, C. S. (1982). The search for school climate: A review of the research. Review of Educational Research, 52 (3), 368-420.

Böhm-Kasper, O. (2004). Schulische Belastung und Beanspruchung. Münster: Waxmann.

Brown, K. E. \& Medway, F. J. (2007). School climate and teachers beliefs in a school effectively serving poor South Carolina (USA) African-American students: A case study. Teaching and Teachers Education: An International Journal of Research and Studies, 23 (4), 529-540.

Bryk, A. S., Sebring, P. B., Allensworth, E., Luperscu, S. \& Easton, J. O. (2010). Organizing schools for improvement. Chicago: The University of Chicago Press.

Cohen, J. (1988). Statistical Power Analysis for the Behavioral Sciences. (Zweite Auflage). Hillsdale: Lawrence Erlbaum.

Cohen, J. \& Geier, V. K. (2010). School climate research summary. New York: Center for Social and Emotional Education.

Cohen, J., McCabe, E. M., Michelli, N. M. \& Pickeral, T. (2009). School climate: Research, policy, practice and teacher education. Teachers College Record, 111 (1), 180-213.

Eder, F. (1996). Was das (Unterrichts-) Klima ausmacht. Pädagogische Führung, 7 (4), $180-183$.

Eder, F. (2001). Schul- und Klassenklima. In D. H. Rost (Ed.), Handwörterbuch Pädagogische Psychologie (S. 578-586). Weinheim: Psychologie Verlagsunion.

Eder, F. (2002). Unterrichtsklima und Unterrichtsqualität. Unterrichtswissenschaft 30 (3), 213-229.

Eder, F. \& Haider, G. (2012). Schulqualität und Befinden im Kontext des familiären Hintergrunds der Schülerinnen und Schüler. In F. Eder (Hrsg.), PISA 2009. Nationale Zusatzanalysen für Österreich. Münster: Waxmann.

Epstein, J. L. \& McPartland, J. M. (1976). The concept and measurement of the quality of school life. American Educational Research Journal, 13 (1), 15-30.

Fend, H. (1986). «Gute Schulen - schlechte Schulen». Die einzelne Schule als pädagogische Handlungseinheit. Deutsche Schule, 78 (3), 275-293.

Fend, H. (1998). Qualität im Bildungswesen. Schulforschung zu Systembedingungen, Schulprofilen und Lehrerleistung. Weinheim: Juventa.

Freiberg, H. J. (1998). Measuring school climate: Let me count the ways. Educational Leadership, 56 (1), 22-26.

Goddard, R. D., Hoy, W. K. \& Hoy, A. W. (2000). Collective teacher efficacy: Its meaning, measure, and impact on students' achievement. American Educational Research Journal, $37(2), 479-507$.

Good, T. L. \& Weinstein, R. S. (1986). Schools make a difference. Evidence, criticisms, and new directions. American Psychologist, 41 (10), 1090-1097.

Gottfredson, G. D., Gottfredson, D. C., Payne, A. A. \& Gottfredson, N. C. (2005). School climate predictors of school disorder: Results from National Study of Delinquency 
Prevention in Schools. Journal of Research in Crime and Delinquency, 42 (4), 412-444.

Halpin, A. W. \& Croft, D. B. (1963). The organizational climate of schools. Chicago, WI: Midwest Administration Center of the University of Chicago.

Holtappels, H. G. \& Voss, A. (2006). Organisationskultur und Lernkultur - Über den Zusammenhang von Schulorganisation und Unterrichtsgestaltung am Beispiel selbstständiger Schulen. In W. Bos, H. G. Holtappels, H. Pfeiffer, H.-G. Rolff \& R. Schulz-Zander (Hrsg.), Jahrbuch der Schulentwicklung, Band 14. Daten, Beispiele und Perspektiven (S. 247-275). Weinheim und München.

Hox, J. J. (2010). Multilevel analysis. Techniques and applications. (Second edition). New York \& Hove: Routledge.

Hoy, W. K. \& Woolfolk, A. E. (1993). Teachers' sense of efficacy and the organizational health of schools. The Elementary School Journal, 93 (4), 356-372.

Johnson, B., Stevens, J. J. \& Zvoch, K. (2007). Teachers' perceptions of school climate: A validity study of scores from the revised School Level Environment Questionnaire. Educational and Psychological Measurement, 67 (5), 833-844.

Johnson, W. L., Johnson, A. M. \& Zimmermann, K. (1996). Assessing school climate priorities: A Texas study. The Clearing House, 70 (2), 64-66.

Keefe, J. W., Kelley, E. A. \& Miller, S. K. (1985). School climate: clear definitions and a model for a large setting. National Association of Secondary School Principial. NASSAP Bulletin, 69, 70-77.

Kuperminc, G. P., Leadbeater, B. J. \& Blatt, S. J. (2001). School social climate and individual differences in vulnerability to psychopathology among middle school students. Journal of School Psychology, 39 (2), 141-159.

Kuperminc, G. P., Leadbeater, B. J., Emmons, C. \& Blatt, S. J. (1997). Perceived school climate and difficulties in the social adjustment of middle school students. Applied Developmental Science, 1 (2), 76-88.

LeBlanc, L., Swisher, R., Vitaro, F. \& Tremblay, R. E. (2007). School social climate and teachers' perceptions of classroom behavior problems: a 10 years longitudinal and multilevel study. Social Psychology of Education, 10 (4), 429-452.

Manning, M. L. \& Saddlemire, R. (1996). Developing a sense of community in secondary schools. National Association of Secondary School Principals. NASSAP Bulletin, 80 (584), 41-48.

Perry, A. (1908). The management of a city school. New York: Macmillan.

Samdal, O., Nutbeam, D., Wold, B. \& Kannas, L. (1998). Achieving health and educational goals through schools - a study of the importance of the school climate and the students' satisfaction with school. Health Education Research, 13 (3), 383-397.

Steinberg, M., Allensworth, E. \& Johnson, D. W. (2011). Students and teacher safety in Chicago public schools: The roles of community context and school social organization. Chicago, WI: Consortium on Chicago School Research.

Stockard, J. \& Mayberry, M. (1992). Effective educational environments. Newbury Park, CA: Corwin Press Inc.

Sutherland, F. (1994). Teachers'perception of school climate. Chicago: Chicago State University.

Sweetland, S. R. \& Hoy, W. K. (2000). School characteristics and educational outcomes: Toward an organizational model of student achievement in middle schools. Educational Administration Quarterly, 36 (5), 703-729.

Way, N., Reddy, R. \& Rhodes, J. (2007). Students` perceptions of school climate during the middle school years: associations with trajectories of psychological and behavioral adjustment. American Journal of Community Psychology, 40 (3), 194-213.

Weick, K. E. (1976). Educational organizations as loosely coupled systems. Administrative Science Quarterly, 21, 1-19.

Weick, K. E. (1982). Administering education in loosely coupled schools. Phi Delta Kappa, 63 (10), 673-676. 
Whitlock, J. L. (2006). Youth perceptions of life at school: Contextual correlates of school connectedness in adolescence. Applied Developmental Science, 10 (1), 13-29.

Zullig, K. J., Koopman, T. M., Patton, J. M. \& Ubbes, V. A. (2010). School climate: Historical review, instrument development, and school assessment. Journal of Psychoeducational Assessment, 28 (2), 139-152.

Schlagworte: Schulklima, Unterrichtsklima, Arbeitsklima, Mehrebenenanalyse

\section{Impact des différences sociales sur les climats relationnel, éducatif et organisationnel dans dans l'école obligatoire en Suisse}

\section{Ré sumé}

Utilisant les données de l'inspection scolaire et de l'Office de la statistique du canton Argovie, la présente étude transversale vise à démontrer l'impact des différences sociales sur les perceptions des élèves à propos du climat relationnel et du climat éducatif ainsi que sur les perceptions des enseignants concernant le climat organisationnel. Suite à une analyse exploratoire factorielle, une analyse multiniveaux a permis d'expliquer la variance en deux niveaux hiérarchiques, soit le niveau individuel du climat éducatif et le niveau collectif de l'établissement scolaire. Les résultats de l'analyse permettent de constater d'une part, que l'inégalité sociale influence significativement le climat relationnel et organisationnel et d'autre part, qu'il est possible pour les enseignants de "faire une différence» dans la classe pour améliorer le climat éducatif.

Mots-clés: Climat relationnel, climat éducatif, climat organisationnel, analyse multiniveaux

\section{Consequenze delle disparità sociali sul clima scolastico, di apprendimento e di lavoro nella scuola obbligatoria Svizzera}

\section{Riassunto}

Basandosi su dati del gruppo di ispezione scolastica e dell'ufficio statistico del cantone Argovia, il presente studio esamina l'impatto delle disparità sociali sul clima scolastico, di apprendimento e di lavoro nell'ambito della scuola obbligatoria Svizzera. In seguito ad analisi preliminari correlative di carattere esplorativo, viene effettuata un analisi multilivello al fine di spiegare la variabilità del clima scolastico di apprendimento. A livello gerarchico di aggregazione viene introdotto un modello di equazioni strutturali che stima l'importanza dei legami tra la variabile esplicativa «indice di disparità sociale» e i modulatori «atmosfera scolastica» e "clima lavorativo degli insegnanti». I risultati dimostrano un notevole impatto delle disparità sociali sul clima scolastico e lavorativo, ma 
non rivelano alcuna evidenza dell'influenza di tali disparità sul clima di apprendimento in classe, suggerendo che questo sia determinato principalmente dagli insegnanti.

Parole chiave: Clima scolastico, clima di apprendimento, clima di lavoro, analisi multivariata

\section{The impact of social deprivation on school climate, learning climate and work climate of compulsory schools in Switzerland}

\section{Summary}

Based on cross-sectional school inspection data and official statistics of the Canton Aargau, the study examines the impact of social deprivation on different aspects of the climate of compulsory schools in Switzerland. Starting with an exploratory correlation of climate factors, the article presents the results of a multi-level analysis with the learning climate as dependent variable on the individual level and a path model on the aggregation level which encompasses the index of social deprivation as independent variable and both the school climate and the work climate of the teaching staff as intermediating variables. The analysis shows a relatively strong impact of social deprivation on school and work climate. The learning climate, however, is not determined by the social environment, which suggests that teachers can make an important difference.

Keywords: School climate, classroom climate, work climate, multi-level analysis 